\title{
The role of stress and age in spatial navigation deficits following prenatal exposure to ethanol
}

\author{
BETTY A. BLANCHARD, MICHELLE L. PILATI, and JOHN H. HANNIGAN \\ State University of New York, Albany, New York
}

\begin{abstract}
The effects of prior experience with swimming on performance in a Morris water task were examined in 21- and 24-day-old rats whose dams consumed liquid diets containing either $35 \%$ or 0\% ethanol-derived calories (EDC) on Days 6-20 of pregnancy, or ad-lib lab chow and water (LC) throughout pregnancy. At 21 days of age, prenatal alcohol-exposed $(35 \% \mathrm{EDC})$ rats displayed impaired performance relative to pair-fed (0\%EDC) and chow-fed (LC) control groups, regardless of prior swimming experience. At 24 days of age, the performance of 35\% EDC rats not given swimming experience prior to training did not differ from controls, which suggests that some recovery from fetal alcohol effects had occurred. Among groups given prior swimming experience, however, the 35\%EDC rats took longer than the 0\%EDC or the LC control groups to find the platform in the Morris maze at 24 days of age. The results suggest that in utero ethanol exposure produces a transient deficit in spatial navigation abilities at around 21 days of age, but that animals appear to recover by around 24 days of age. This deficit appeared to be revealed in 24-day-old rats by giving the animals swim experience prior to training. The results suggest that the present and similar deficits and in spatial navigation in prenatal alcohol-exposed rats may be due to increased and/or prolonged stress responses to the task in alcohol-exposed offspring, and not to a hippocampus-mediated deficit in spatial navigation per se.
\end{abstract}

Chronic alcohol consumption during pregnancy can produce fetal alcohol syndrome (FAS) in the offspring, which is characterized by morphological and functional abnormalities (Jones \& Smith, 1973). Alcohol-related developmental effects observed in the absence of full expression of FAS include hyperactivity and learning deficits (Streissguth, 1986). Animal models of fetal alcohol effects have produced behavioral deficits similar to those seen in humans, such as increased open-field activity and poor learning (Meyer \& Riley, 1986).

Among the neuroanatomical anomalies in rodents exposed perinatally to ethanol are a decreased number of hippocampal pyramidal cells (Barnes \& Walker, 1981), altered dendritic branching and a decreased number of dendritic spines on hippocampal pyramidal cells (Abel, Jacobson, \& Sherwin, 1983), and aberrant mossy fiber innervation of CA3 (West, Hodges, \& Black, 1981). These hippocampal anomalies may be of functional significance, since animals exposed prenatally to alcohol display a number of behavioral similarities to animals with hippocampal lesions. Ethanol-exposed and hippocampal-lesioned animals both show increased open-field activity, spontaneous alternation deficits, and poor passive avoidance performance (for review, see Riley, Barron, \& Hannigan,

This research was funded by Grants AA06721 and AA00111 from the National Institute of Alcoholism and Alcohol Abuse to J.H.H. A preliminary report was made to the annual meeting of the International Society for Developmental Psychobiology, Toronto, Ontario, Canada, November 1988. Address correspondence to J. H. Hannigan, Center for Behavioral Teratology, State University of New York, SS-112, 1400 Washington Avenue, Albany, NY 12222.
1986). On the other hand, fetal ethanol-exposed rats display an enhanced grooming response to novel, stressful environmental stimuli (Hannigan, Blanchard, \& Riley, 1987), whereas animals with hippocampal lesions show attenuated novelty-induced grooming (Reinstein, Hannigan, \& Isaacson, 1982). Moreover, alcohol-exposed rats exhibit two-way avoidance deficits, while hippocampal-lesioned animals perform better than controls (Riley et al., 1986).

The hippocampus plays an important role in spatial learning and memory (O'Keefe \& Nadel, 1978; Olton, Becker, \& Handelmann, 1979). To clarify hippocampal involvement in fetal alcohol effects on behavior, we had previously examined the performance of fetal ethanolexposed rats on the Morris water task (Blanchard, Riley, \& Hannigan, 1987), a spatial learning task sensitive to hippocampal dysfunction (Morris, 1981; Morris, Garrud, Rawlins, \& O'Keefe, 1982). Rats placed into a circular pool of opaque water learn to escape by locating and climbing onto a platform hidden below the surface of the water. Normal animals perform this task quite well by learning the location of the platform relative to visible cues surrounding the pool, as indicated by their preference for searching in the original goal quadrant when the platform is removed for a probe trial. Hippocampal-lesioned rats take longer to learn to reach the platform, travel more circuitous paths, and show no preference for the goal quadrant when the platform is removed during a probe trial (Morris et al., 1982).

We reported previously that between Postnatal Day 22 (PN22) and PN24, rats exposed prenatally to alcohol took longer than controls to learn to locate the platform in this task, and that the deficit appeared to be slightly more 
pronounced in females than in males (Blanchard, Riley, \& Hannigan, 1987). Goodlett, Kelly, and West (1987) reported that rats exposed neonatally to ethanol during a period of rapid hippocampal development also show deficits on the Morris water task. These findings suggest that perinatal alcohol exposure may disrupt hippocampal mechanisms involved in spatial learning in rats. There are, however, several alternative explanations of the deficit. For example, fetal ethanol exposure may produce attention deficits (Strupp, Korahais, Levitsky, \& Ginsberg, 1989). If so, then prenatal ethanol-exposed rats may simply require more salient visual information to engage a spatial search strategy than do controls. We examined this possibility by manipulating the degree to which visual cues were present (Blanchard, Hannigan, \& Riley, 1987), and found that with enhanced visual cues, fetal ethanolexposed animals navigate as well as controls, on the basis of both latency data and probe-trial data. However, when access to visual cues was severely restricted, fetal ethanolexposed rats took longer than controls to reach the platform. Interestingly, when the platform was removed for a probe trial after restricted cue training, search patterns indicated no preference for the goal quadrant in any prenatal treatment group. Controls were apparently able to develop an effective nonmapping strategy in the absence of obvious visual cues that allowed them to locate the platform as quickly as when cues were present. However, alcohol-exposed animals were unable to do so. These results suggested to us that the deficit is not spatial per se, but that fetal ethanol-exposed animals may be deficient in their ability to develop effective localization strategies in situations in which spatial information is scarce or unavailable.

Another possibility is that Morris water task deficits may be related to stress-induced performance deficits rather than long-term learning deficits. Fetal ethanolexposed rats show enhanced stress responses to a variety of stimuli (Taylor, Nelson, Branch, Kokka, \& Poland, 1984), including increased stress-induced grooming responses to novel, forced swimming (Hannigan et al., 1987). We also reported that prenatal ethanol-exposed rats were more likely than controls to exhibit grooming behavior while on the platform in a Morris task (Blanchard, Riley, \& Hannigan, 1987), which suggests that they were indeed more stressed than controls. In the present study, we examined the role of stress in spatial deficits in fetal ethanol-exposed animals by manipulating the degree to which swimming was a novel experience. It has been shown that decreasing swim novelty by pretraining exposure to swimming attenuates Morris water task deficits in neonatally malnourished rats (Goodlett, Valentino, Morgane, \& Resnick, 1986). In the present study, we assessed whether a similar pretraining exposure to swimming would alleviate Morris water task deficits in fetal ethanol-exposed animals.

Finally, Morris water task deficits in fetal ethanolexposed rats at the ages tested (PN22-PN24) may be due to an alcohol-related delay in the development of spatial learning. Normal animals begin to exhibit spatial navigation abilities around PN20 to PN22 (Rudy, StadlerMorris, \& Albert, 1987; Schenck, 1985). The improved performance we observed by Day 3 of testing (PN24) in our previous study (Blanchard, Riley, \& Hannigan, 1987) may have been due to later hippocampal maturation in alcohol-exposed progeny. A delay has been reported in the development of mouse hippocampal pyramidal cells following perinatal alcohol exposure (Davies \& Smith, 1981). Therefore, in the present study, we began training animals at either PN21 or PN24 to examine the role of development in fetal ethanol-induced spatial navigation deficits.

\section{EXPERIMENT 1}

\section{Method}

Procedures for generating alcohol-exposed offspring are detailed elsewhere (Hannigan et al., 1987). Pregnant Long-Evans hooded rats (Blue Spruce Farms, Altamont, NY) were housed individually and assigned randomly to one of three treatment groups. One group received ad-lib access to a liquid diet containing $35 \%$ ethanol-derived calories (35\%EDC) on Days 6-20 of pregnancy. A second group was pair-fed the liquid diet with sucrose substituted isocalorically for ethanol (0\%EDC) and served as a yoked control for the effects of the liquid diet. A third group received ad-lib lab chow and water throughout pregnancy (LC). Liquid diets consisted of water, chocolate Sustacal (Mead Johnson, Inc.), Vitamin Diet Fortification Mixture, and Salt Mixture (ICN Nutritional Biochemicals), with either $95 \%$ ethanol or sucrose added. Liquid diets provided approximately $1.3 \mathrm{kcal} / \mathrm{ml}$ and were the sole source of nutrition during the period they were administered.

Pups were weighed on the day following birth (Day 1), inspected for malformations, and the litters were culled to 10 pups, maintaining 5 males and 5 females whenever possible. Pups remained with their dams until weaning at PN21, when they were singly housed and maintained on a 12:12 h light:dark cycle (lights on at $0700 \mathrm{~h}$ ) with food and water available throughout.

Behavioral testing. The experimental procedure consisted of exposing some animals to swimming on 2 consecutive days beginning on PN22. All animals were then trained for 3 days in a Morris maze, beginning on PN24. On each of these days, the animals were tested with a probe trial following the training session. On the day following the last training session, the animals received only a probe trial. Male and female rats from the three prenatal treatment groups served as subjects ( $n \mathrm{~s}=6-7$ per cell). No more than 1 rat per litter was used in any cell of the design, in order to control for possible litter effects. All training and testing took place between 0800 and $1200 \mathrm{~h}$.

Initial pre-exposure to swimming took place in a 40 -liter aquarium $\left(26^{\circ} \mathrm{C}\right)$. Half of the animals from each of the prenatal treatment groups were placed into the aquarium and allowed to swim freely for $1 \mathrm{~min}$ on each of 2 consecutive days (PN22 and PN23). The other animals remained in their home cages until training began on PN24 for all groups.

The aquarium and the Morris maze were located in different rooms. The maze was a circular tank (100-cm diam., $45-\mathrm{cm}$ high) filled with opaque water ( $8 \mathrm{~cm}$ from top of tank) and surrounded by a number of visual cues, which included items such as a bookshelf, a small table, a poster, and an overhead video camera. The tank was divided into four quadrants (indistinguishable by the rats). A platform (10-cm diam.) was placed near the center of one of the 
quadrants and remained in the same location throughout training for each animal. A training trial consisted of placing a rat into one of the three other quadrants facing outward, and allowing it to swim until it reached and climbed onto the platform, or until 2 min elapsed without the rat reaching the platform. Animals that did not locate the platform within 2 min were placed on the platform by the experimenter. The animals remained on the platform for $15 \mathrm{sec}$ before being removed. No animal was placed at the same starting point on consecutive training trials. Each rat received 10 trials per day for 3 days.

The animals were tested in squads of 4 . The intertrial interval (ITI) varied depending on the latency scores of the animals in the squad (range: $240-600 \mathrm{sec}$ ). ITIs were longer early in training, and decreased over trials. There was no evidence that squads with different ITIs performed differently in the Morris maze. Following training on each day, the platform was removed and the animal was placed into the center of the tank for a $30-\mathrm{sec}$ probe trial. There was also a final probe trial on Day 4 (PN27). Probe trials were videotaped and later scored using an event recorder (S \& K Electronics, Toronto) to measure time spent in each quadrant. The dependent measures were latency to reach the platform during training and the proportion of time spent in each quadrant during the probe trials.

\section{Results}

Maternal data. Maternal data were analyzed by analyses of variance (ANOVAs) with diet as the factor. There was a significant effect of diet on percentage of weight gain during pregnancy $[F(2,85)=4.38, p<.05]$. NewmanKeuls comparisons $(p<.05)$ indicated that the LC dams gained more weight during pregnancy than the $35 \%$ EDC and $0 \%$ EDC dams, which did not differ from each other. Diet did not affect gestation length, litter size, or sex ratio of the litters.
Pup data. Pup data were analyzed by ANOVA, with prenatal treatment and sex as factors. There was a significant effect of prenatal treatment on birth weight $[F(2,170)=59.63, p<.0001]$. Comparisons indicated that $35 \%$ EDC pups weighed less than LC and 0\%EDC pups, which did not differ from each other. Males weighed more than females at birth $[F(1,170)=18.86, p<.0001]$, regardless of prenatal treatment.

Training (Days 1-3). Latency scores were totaled for each of six five-trial blocks. These scores were then analyzed by ANOVA, with prenatal treatment, sex, and pretraining exposure condition as between-subjects factors, and block within day as a nested within-subjects factor. There was a significant treatment $\times$ condition $\times$ block $X$ day interaction $[F(4,234)=2.78, p<.05]$. NewmanKeuls comparisons $(p<.05)$ indicated that although the $35 \%$ EDC rats in the nonexposed condition performed as well as the controls, the alcohol-exposed rats that received prior exposure to swimming in the aquarium took longer than the controls to locate the platform over the first three blocks (Figure 1). All animals showed shorter latencies as training progressed [main effect of Day, $F(2,122)=$ 199.07, $p<.0001$ ]. LC and 0\%EDC animals did not differ from each other in either condition.

Probe trials. The proportion of time spent in each quadrant for the four probe trials was subjected to an arc sine transformation (Winer, 1971) and analyzed by ANOVA, with prenatal treatment, sex, and pretraining exposure condition as between-subjects factors, and quadrant and day as within-subjects factors. A significant main effect of quadrant $[F(3,183)=125.04, p<.0001]$ was due to

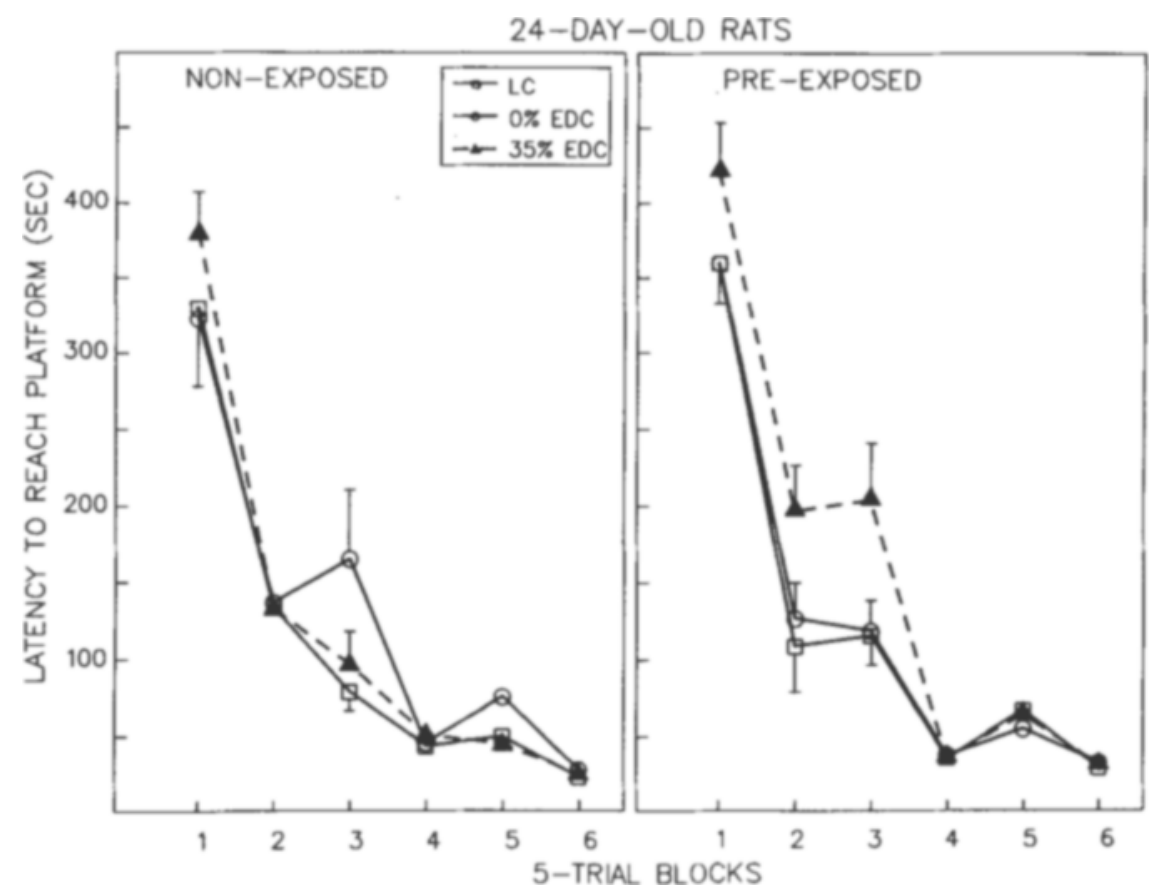

Figure 1. Mean ( $\pm S E M$ ) latencies for 24-day-old rats from each prenatal treatment group for nonexposed and pre-exposed swim conditions collapsed across sex for each five-trial block in Experiment 1. Prenatal alcohol-exposed offspring in the prior swim-experience condition took longer than controls to reach the platform on the first three blocks (Trials 1-15). 
the fact that all groups spent a greater proportion of time in the goal quadrant than in any of the other quadrants. A significant quadrant $\times$ day interaction $[F(9,549)=8.03$, $p<.0001]$ was due to the fact that the animals showed successive increases in the proportion of time spent in the goal quadrant on Days 2 and 3, then a decrease on Day $4(p<.05)$. Prenatal treatment had no effect on quadrant preference. Nontransformed data are presented in Figure 2.

\section{EXPERIMENT 2}

Twenty-four-day-old prenatal alcohol-exposed animals in Experiment 1 without prior exposure to swimming in the aquarium did not show the spatial navigation deficits that we had reported previously (Blanchard, Hannigan, \& Riley, 1987; Blanchard, Riley, \& Hannigan, 1987). Because the rats in the current study were slightly older than the animals we had tested previously (PN24 vs. PN22 at the start of training), it may be that fetal alcohol exposure results in a delay in the development of spatial navigation abilities from which the animals in Experiment 1 had recovered. Therefore, in Experiment 2 we gave animals experience with swimming on PN19 and PN20 and began training on PN21 to determine if the deficit was present at that younger age. Breeding, pretraining swimming exposure, training, and testing procedures were identical to those described in Experiment 1, except that the animals were weaned on PN19. Maternal and pup characteristics were similar to those in Experiment 1.

\section{Results}

Training (Days 1-3). Latency data (Figure 3) were analyzed as described in Experiment 1 . Independent of pretraining exposure condition and sex, the $35 \%$ EDC rats took longer than the controls to reach the platform throughout training [prenatal treatment, $F(2,56)=4.55, p<.05$; see Figure 3 inset]. All animals showed improved performance over days $[F(2,112)=159.90, p<.0001]$.

Probe trials. Proportion data for the probe trials were transformed and analyzed as described in Experiment 1. There was a significant prenatal treatment $x$ quadrant $x$ day interaction $[F(18,504)=1.70, p<.05]$. Comparisons indicated that the LC animals showed a significant preference for the goal quadrant on each probe trial. However, on Day 1, the 0\%EDC and 35\%EDC animals showed no preference for the goal quadrant. On Day 2, the 0\%EDC and $35 \% \mathrm{EDC}$ rats did prefer the goal quadrant, although the $35 \% \mathrm{EDC}$ rats spent significantly less time there than did the LC controls. The pair-fed $(0 \%$ EDC) rats did not differ from either of the other groups. On Days 3 and 4 , there were no group differences in the proportion of time spent in the goal quadrant. Figure 4 represents nontransformed data.

\section{DISCUSSION}

The results of Experiment 1 indicated that the Morris water task performance of 24-day-old fetal ethanolexposed rats is sensitive to behavioral manipulation prior to training. When prenatal alcohol-exposed (35\% EDC)

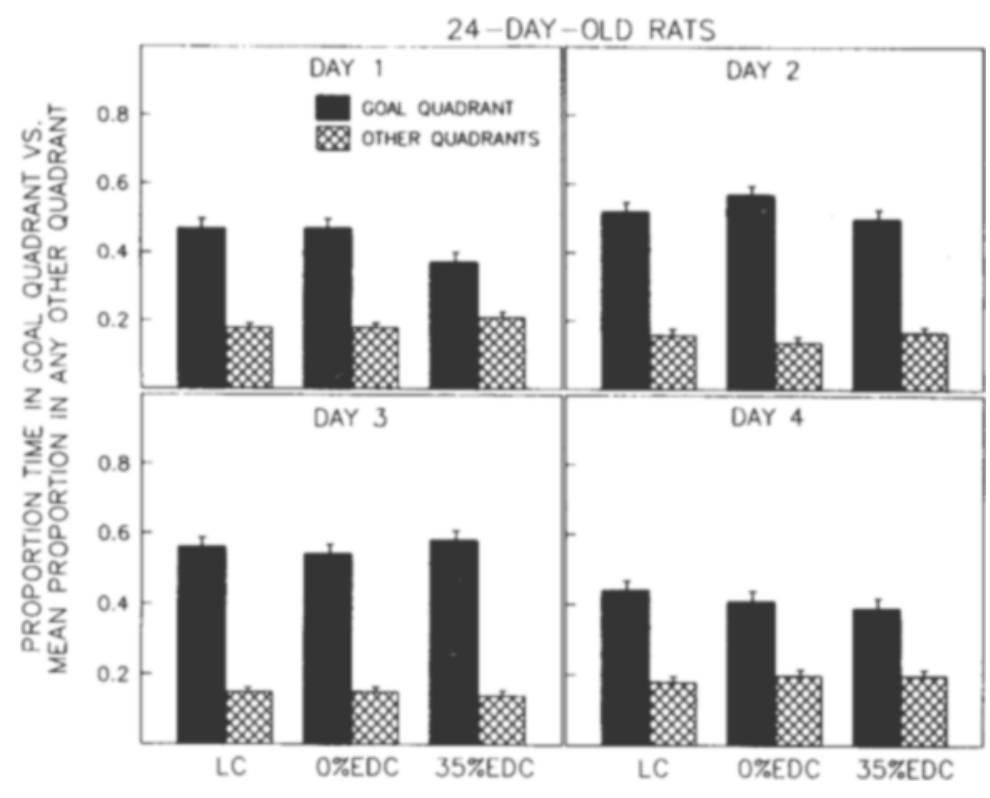

Figure 2. Probe-trial quadrant preferences for 24-day-old rats from each prenatal treatment group in Experiment 1 collapsed across sex and swim-exposure condition. The graphs represent the mean $( \pm S E M)$ proportion of time spent in the goal quadrant and in any of the other three quadrants. All groups spent increasing amounts of time in the goal quadrant on each probe trial. 


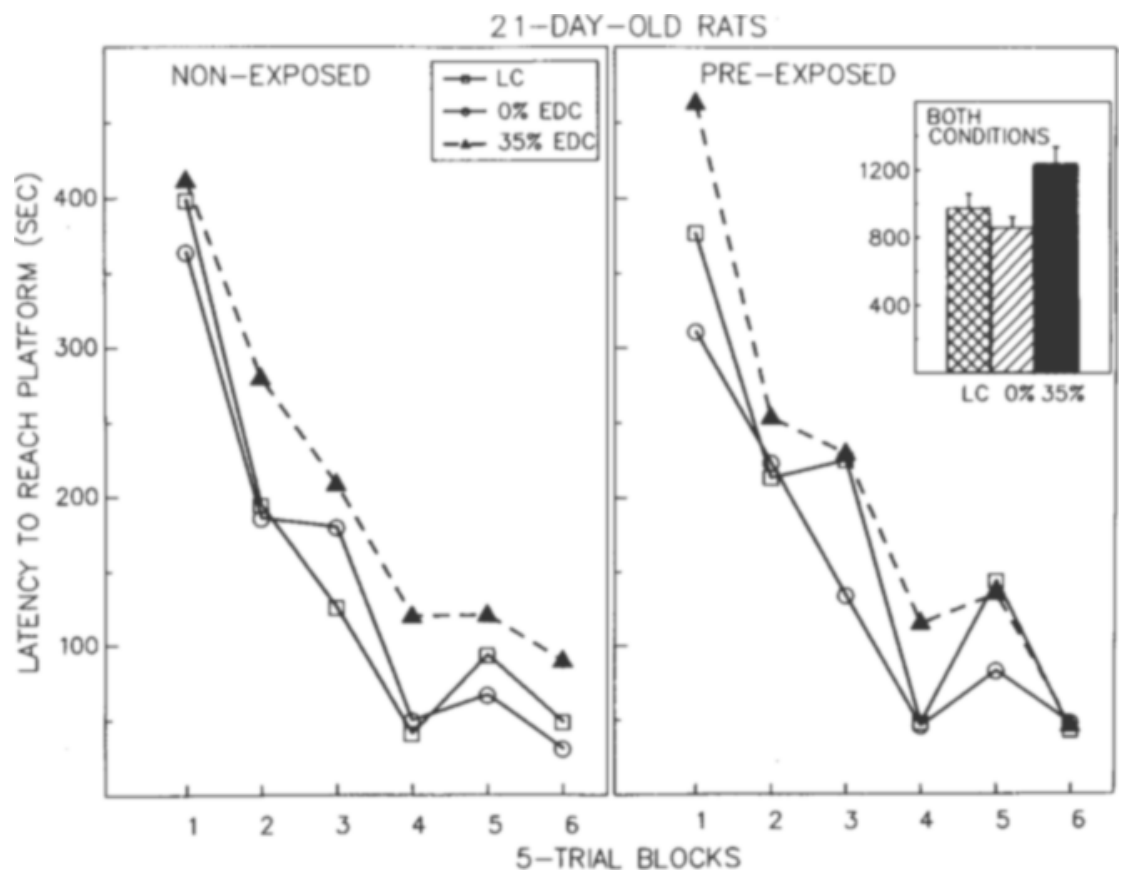

Figure 3. Mean latencies for 21-day-old rats from each prenatal treatment group for nonexposed and pre-exposed swim conditions collapsed across sex for each five-trial block in Experiment 2. The significant main effect of prenatal treatment is depicted in the bar graph inset, which represents mean ( $+S E M)$ total latency collapsed across sex, swim-exposure condition, and block. Overall, prenatal alcoholexposed animals took longer than controls to reach the platform.

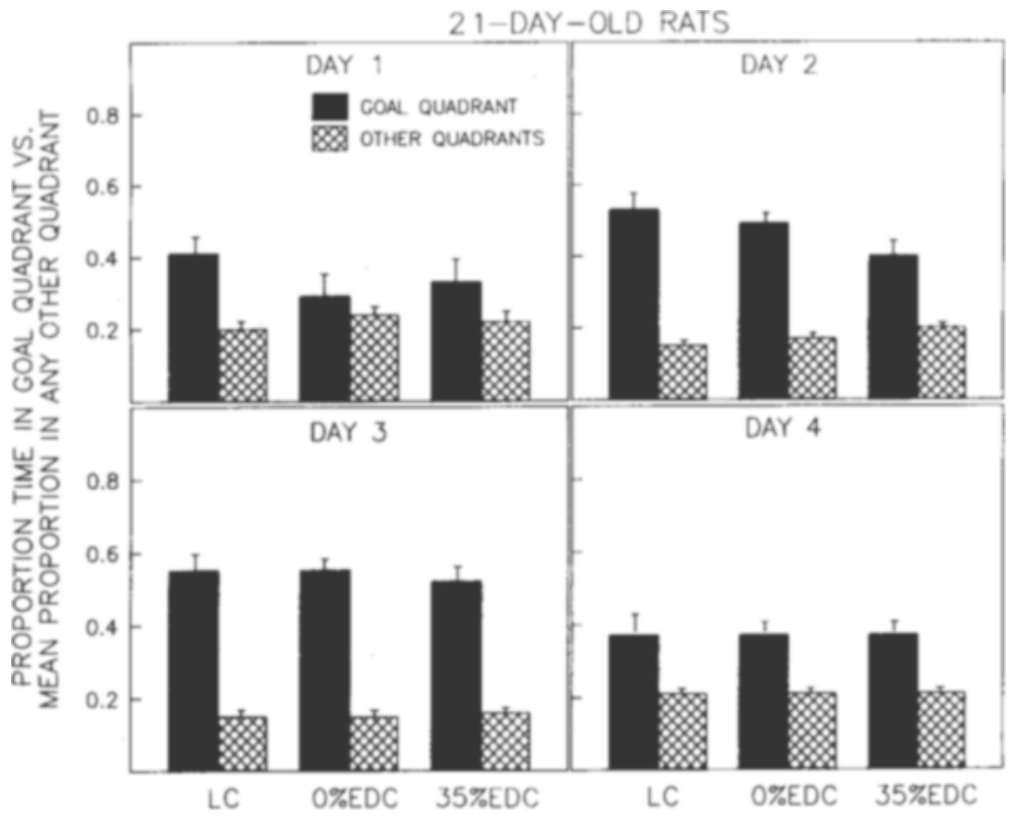

Figure 4. Probe-trial quadrant preference for 21-day-old rats from each prenatal treatment group in Experiment 2 collapsed across sex and prior swim-exposure condition. The graphs represent the mean $(+S E M)$ proportion of time spent in the goal quadrant and in any of the other three quadrants. On Day 1 , the prenatal alcohol-exposed (35\%EDC) and nonexposed (0\%EDC) groups showed no significant preference for the goal quadrant. On all other probe trials, all groups preferred the goal quadrant. 
animals were not given swimming experience before training, their performance on this task was comparable to that of controls. However, when the $35 \%$ EDC animals were exposed to swimming prior to training, they showed a latency deficit early in training, but did not show a deficit in search patterns on any of the probe trials. The finding of latency deficits, but normal search patterns on probe trials, is not likely to be due to differences in swimming speed among the prenatal treatment groups, since (1) we examined swimming speed in two previous experiments and found no effect of prenatal alcohol, and (2) the $35 \%$ EDC animals had longer latencies only in the pretraining exposure condition. The normal performance on probe trials by the $35 \% \mathrm{EDC}$ rats despite latency deficits during training may be related to the fact that by the end of the training session, on any given day the performance of the $35 \% \mathrm{EDC}$ rats in the preswim condition approached that of the controls. That is, although there were overall latency deficits on Day 1 and on the first five-trial block on Day 2, latency scores of the 35\%EDC rats in the pretraining exposure condition were comparable to those of controls for the last trial of each session. Differences in search patterns might have been detected in those animals if there had been a longer interval between the last training trial and the probe trial. This is suggested by the fact that the $35 \% \mathrm{EDC}$ rats in the pretraining exposure condition, which showed latency scores similar to those of controls on the last training trial of Day 1, showed latency deficits again on the first five-trial block on Day 2.

In Experiment 2, 21-day-old prenatal ethanol-exposed animals took longer than controls to locate the platform over the entire training period, regardless of prior swimming exposure. Both the $35 \%$ EDC and the $0 \%$ EDC rats showed no preference for the goal quadrant until the second probe trial. However, on the second probe trial, the $35 \%$ EDC rats spent less time in the goal quadrant than did the LC controls. In contrast to our earlier report (Blanchard, Riley, \& Hannigan, 1987), there were no significant effects of sex in the present experiments.

The present experiments indicate that spatial navigation deficits are present in 35\%EDC rats around PN21 and that prior exposure to swimming does not affect performance of this task at this age in any group of animals. By PN24 the 35\%EDC rats did not exhibit spatial navigation deficits in the nonexposed condition; however, when 24-day-old rats were exposed to swimming prior to training, a latency deficit was apparent. It may be that fetal ethanol exposure resulted in a delay in the development of spatial navigation, producing a transient deficit that can be reinstated by exposing the animal to swimming prior to training.

This finding is contrary to our prediction that prior swimming experience would attenuate the deficit as such experience had done for similar deficits resulting from neonatal protein malnutrition (Goodlett et al., 1986). One possible explanation for the effects may be an enhanced stress response in alcohol-exposed offspring. In an earlier study, we reported that $35 \%$ EDC rats exposed to a forced 1-min swim showed an increased stress-induced grooming response relative to controls (Hannigan et al., 1987). We also reported that when animals tested in the Morris water test were forced to remain on the platform for $180 \mathrm{sec}, 35 \%$ EDC rats were more likely than controls to groom themselves (Blanchard, Riley, \& Hannigan, 1987). In the present study, grooming during the $15 \mathrm{sec}$ the rats were left on the platform was quite rare and apparently did not differ among groups. If initial exposure to swimming was more stressful to the $35 \% \mathrm{EDC}$ animals than to the controls, this could have produced "sensitization" rather than "acclimation" to the stressful aspects of swimming, which in turn may have interfered with later performance in the Morris maze.

We are aware of no data that suggest that the behavior of prenatal ethanol-exposed animals may become either less habituated or more sensitized to the effects of stress. There are, however, biochemical data that suggest that alcohol-exposed offspring do not respond to procedures that attenuate stress responses in normal animals. Weinberg (1988) recently showed that fetal alcohol-exposed animals had enhanced corticosterone release in response to stress, and that procedures that attenuated corticosterone release after stress in normal animals were not as effective in fetal alcohol-exposed animals. We have no direct data on stress responses of animals in this study.

Alternatives to the stress interpretations of our results include differences in learning not related to spatial navigation per se. For example, at 24 days of age, prenatal alcohol-exposed rats might not benefit from learning general aspects about swimming during their prior experience. However, there was no evidence of such a benefit in controls since there were no differences between groups with or without prior swimming experience. Another possibility is that during the prior swim experience, the alcohol-exposed rats learned a particular response, such as swimming until being removed by the experimenter. In such a case, the deficit in the Morris maze could reflect a response-inhibition deficit-that is, perseveration of a previously learned response (cf. Riley et al., 1986). It is also possible that our interpretations about apparent developmental delays may be limited by differences in weaning age between the two experiments. All groups within each experiment were weaned at the same age, and we did not analyze for an age factor (i.e., between experiments). It is possible, however, that prenatal alcohol exposure interacted with age at weaning to affect performance in the Morris maze without causing a developmental delay. Finally, it does not appear that undernutrition alone (the pair-fed groups) substantially influenced performance, although the 21-day-old 0\%EDC group did show a quadrant preference intermediate to the other groups.

The finding that alcohol-exposed offspring without prior swim experience did not show a deficit on the Morris water task by PN24 is in contrast to recent findings that prenatal alcohol-induced deficits on this task persist into adulthood (Gianoulakis, in press). However, in that study, 
the animals were not placed onto the platform if they failed to locate it by the end of a training trial. Since our methods included this correction procedure, the present task may have been easier to master.

In summary, by our procedure, fetal ethanol-induced spatial navigation deficits at around 21 days of age appear to reflect a developmental delay rather than a longlasting deficit in spatial ability per se. Since impaired performance can be reinstated at 24 days of age by exposing the animal to swimming prior to training, the deficit at both ages may reflect abnormal biobehavioral responses to stress rather than mechanisms controlling spatial navigation per se. Our findings suggest that even in animals that appear to have recovered from fetal ethanol effects, learning can be compromised by stressful exposure to novel stimuli. However, other procedures (e.g., different levels or patterns of ethanol exposure and/or more difficult spatial tasks) may reveal more specific and/or more persistent spatial deficits.

\section{REFERENCES}

Abel, E. L., JACobson, S., \& SHerwin, B. T. (1983). In utero alcohol exposure: Functional and structural brain damage. Neurobehavioral Toxicology \& Teratology, 5, 363-366.

Barnes, D. E., \& WALKer, D. W. (1981). Prenatal ethanol exposure permanently reduces the number of pyramidal cells in rat hippocampus. Developmental Brain Research, 1, 333-340.

Blanchard, B. A., Hannigan, J. H., \& Riley, E. P. (1987). Prenatal alcohol exposure disrupts spatial localization strategies in young rats. Alcoholism: Clinical \& Experimental Research, 11, 222. (Abstract No. 177)

Blanchard, B. A., Ruley, E. P., \& Hannigan, J. H. (1987). Deficits on a spatial navigation task following prenatal exposure to ethanol. Neurobehavioral Toxicology \& Teratology, 9, 253-258.

Davies, D. L., \& SмITh, D. E. (1981). A Golgi study of mouse hippocampal CAl pyramidal neurons following perinatal alcohol exposure. Neuroscience Letters, 26, 49-54.

GianoulaKIs, C. (in press). Rats exposed prenataily to alcohol exhibit impairment in a spatial navigation test. Behavioral Brain Research.

Goodlett, C. R., Kelly, S. J., \& West, J. R. (1987). Early postnatal alcohol exposure that produces high blood alcohol levels impairs development of spatial navigation learning. Psychobiology, 15, 64-74.

Goodlett, C. R., Valentino, M. L., Morgane, P. J., \& Resnick, O. (1986). Spatial cue utilization in chronically malnourished rats: Taskspecific learning deficits. Developmental Psychobiology, 19, 1-16.

Hannigan, J. H., Blanchard, B. A., \& Riley, E. P. (1987). Altered grooming responses to stress in rats exposed prenatally to ethanol. Behavioral \& Neural Biology, 47, 173-185.
Jones, K. L., \& SMITh, D. W. (1973). Recognition of the fetal alcohol syndrome in early infancy. Lancet, 2, 999-1001.

Meyer, L. S., \& Riley, E. P. (1986). Behavioral teratology of alcohol. In E. P. Riley \& C. V. Voorhees (Eds.), Handbook of behavioral teratology (pp. 101-140). New York: Plenum.

Morris, R. G. M. (1981). Spatial localization does not require the presence of local cues. Learning \& Motivation, 12, 239-260.

Morris, R. G. M., Garrud, P., Rawlins, J. N., O'Keefe, J. (1982). Place navigation is impaired in rats with hippocampal lesions. $\mathrm{Na}$ ture, 297, 681-683.

O'KeEFE, J., \& NADEL, L. (1978). The hippocampus as a cognitive map. Oxford: Clarendon.

Olton, D. S., Becker, J. T., \& Handelmann, G. E. (1979). Hippocampus, space and memory. Behavioral \& Brain Sciences, 2, 313-322.

Reinstein, D. K., Hannigan, J. H., \& IsaAcson, R. L. (1982). The time course of certain behavioral changes after hippocampal lesions and their alteration by dopaminergic intervention into nucleus accumbens. Pharmacology, Biochemistry \& Behavior, 17, 193-202.

Riley, E. P., Barron, S., \& Hannigan, J. H. (1986). Response inhibition deficits following prenatal alcohol exposure: A comparison to the effects of hippocampal lesions in rats. In J. R. West (Ed.), Alcohol and brain development (pp. 71-102). New York: Oxford University Press.

Rudy, J. W., Stadler-Morris, S., \& Albert, P. (1987). Ontogeny of spatial navigation behaviors in the rat: Dissociation of "proximal"and "distal"'-cue-based behaviors. Behavioral Neuroscience, 101, 62-73.

SCHENCK, F. (1985). Development of place navigation in rats from weaning to puberty. Behavioral \& Neural Biology, 43, 69-85.

Streissguth, A. (1986). The behavioral teratology of alcohol: Performance, behavioral, and intellectual deficits in prenatally exposed children. In J. R. West (Ed.), Alcohol and brain development (pp. 3-44). New York: Oxford University Press.

StrupP, B. J., KorahaIs, J., Levitsky, D. A., \& GinsBerg, S. (1989). Attentional impairment in rats exposed to alcohol prenatally: Lack of hypothesized masking by food deprivation. In D. E. Hutchings (Ed.), Prenatal abuse of licit and illicit drugs (Vol. 562, pp. 380382). New York: New York Academy of Sciences.

Taylor, A. N., Nelson, L. R., Branch, B. J., Kokka, N., \& Poland, R. E. (1984). Altered stress responsiveness in adult rats exposed to ethanol in utero: Neuroendocrine mechanisms. In Mechanisms of alcohol damage in utero. Ciba Foundation Symposium, 105 , 47-65. London: Pitman.

WEINBERG, J. (1988). Hyperresponsiveness to stress: Differential effects of prenatal ethanol on males and females. Alcoholism: Clinical \& Experimental Research, 12, 647-652.

West, J. R., Hodges, C. A., \& Black, A. C. (1981). Prenatal exposure to ethanol alters the organization of hippocampal mossy fibers in rats. Science, 211, 957-959.

WiNER, B. J. (1971). Statistical principles in experimental design (2nd ed.). New York: McGraw-Hill.

(Manuscript received August 14, 1989; revision accepted for publication January 9, 1990.) 